\title{
The Sexual Addiction Controversy: A Disorder or Not?
}

\author{
Stefany Valentia ${ }^{1, *}$ Elizabeth Kristi Poerwandari ${ }^{2}$
}

\author{
${ }^{1}$ Clinical Psychology Department Faculty of Psychology University of Indonesia \\ ${ }^{2}$ Clinical Psychology Department Faculty of Psychology University of Indonesia \\ *Corresponding author. Email: stefany.valentia@gmail.com
}

\begin{abstract}
The status of sexual addiction as a mental disorder remains controversial. Regardless of the popularity of the term sexual addiction today, the fact is that the proposal of sexual addiction diagnosis has been unclear. This has sparked a debate among clinicians, there are clinicians who support the inclusion of sexual addiction as a mental disorder and clinicians who believe that sexual addiction should not be considered as a mental disorder. This paper aims to examine the evidence and arguments from both sides through literature review, so that it can be concluded whether sexual addiction is an independent mental disorder or not. Based on literature review that has been conducted, it can be concluded that declaring sexual addiction as an independent mental disorder is a statement that is still premature and has potential negative impacts, especially because the scientific boundaries that underlie the diagnosis are still unclear and inconsistent. Another reason is due to the fact that other psychological problems and emotional regulation problems often comorbid in patients who have excessive sexuality problems, so there is a possibility that what we have known as sexual addiction is actually only a manifestation of another mental disorder and is not an independent mental disorder. The contribution of this research is to clarify misunderstanding that happens among clinicians and society about the term sexual addiction, especially about its status that is not classified as a mental disorder.
\end{abstract}

Keywords: Sexual Addiction, Sex Addict, Sexual Impulsivity, Compulsive Sexual Behavior, compulsive sex, excessive sexual behavior, hypersexual, hypersexuality

\section{INTRODUCTION}

To date, the existence of sexual addiction as a psychological disorder remains controversial among clinicians. The amount of movies with the theme of sexual addiction such as "Diary of a Sex Addict", "I Am Sex Addict", "Love Sick: Secrets of a Sex Addict", etc., are increasing and this makes people more often to hear the term sexual addiction and think of it as a psychological disorder. Actually, sexual addiction is not a new concept in which in the ancient Rome and Greece, there had been reports of excessive sexuality that was also known as hypersexuality and nymphomania in women. The modern concept of sexual addiction was first introduced by Dr. Patrick Carnes in his book "Out of the Shadows: Understanding Sexual Addiction" and with his colleagues at a clinic in Arizona. Carnes and his colleagues have written books about sexual addiction and these works have dominated the popular understanding of sexual addiction. In his book, Carnes popularized sexual addiction as a psychopathological condition.
Besides the works of Carnes and his colleagues, there are also other writers who write about the topic of sexual addiction, including researchers and people who believe that they experience sexual addiction. Here are the signs of sexual addiction adapted from Carnes's book [1]:

- Out of control sexual behavior.

- Inability to stop sexual behavior.

- Constant pursuit of high-risk behavior.

- Ongoing desire or effort to limit sexual behavior.

- Sexual behavior (obsession and sexual fantasy) is used as the main coping strategy.

- Presence of tolerance phenomenon.

- Severe mood changes associated with sexual activity.

- $\quad$ Spending excessive time for getting sex. 
- Spending excessive time for being sexual or recovering from sexual experience.

- Severe social, physical and psychological consequences.

The term sexual addiction is often regarded as a disorder, especially because sexual addiction is believed to have negative effects on a person's functioning. However, despite the popularity of the term sexual addiction, the fact is that sexual addiction diagnosis proposal has been refused from DSM-5. The rejection is mainly because of a lack of peer-reviewed evidence to determine the diagnosis criteria [2]. On the other hand, there might be undeniable benefits if sexual addiction is included in DSM-5, such as standardizing the diagnosis among therapists and facilitating treatment plan. Moreover, the existence of a diagnosis makes it easier for clients to request insurance protection.

The rejection from DSM-5 continues the debate in the clinical field, is sexual addiction really a disorder? Is the term "addiction" that has been attached to that set of symptoms the right term? This debate is further complicated when the International Classification of Disease 11 (ICD-11) has accepted excessive sexual behavior as a disorder, but use the term "Compulsive Sexual Behavior Disorder" and include it in the part of impulsive disorder, and not in the part of addiction disorder. So, according to the classification of the ICD, the term "addiction" is a less appropriate term to be used to explain excessive sexual behavior.

Various terms have been used to name the sexual addiction-like condition, including hypersexual disorder, nymphomania, Don Juanism, satyriasis, sexual compulsivity, sexual impulsivity, and uncontrolled sexual behavior. In this paper, the author wants to discuss whether or not sexual addiction is a psychological disorder / mental disorder.

\section{METHOD}

The design used in this paper was a thematic literature review of research articles. Thematic literature review of research articles was done in order to obtain current knowledge that exists around the phenomenon discussed. The articles reviewed were sourced from online database, such as ProQuest, SpringerLink, Sage Publication, Taylor and Francis, and Science Direct via Universitas Indonesia e- library access. The keywords utilized in the searches were sexual addiction controversy, sex addict controversy, sexual impulsivity controversy, compulsive sexual behavior controversy, compulsive sex controversy, excessive sexual behavior controversy, hypersexual controversy, and hypersexuality controversy.
Table 1. Inclusion and Exclusion Criteria

\begin{tabular}{|c|c|}
\hline Inclusion Criteria & Exclusion Criteria \\
\hline $\begin{array}{c}\text { Published between 2006 } \\
\text { and 2018 }\end{array}$ & Published prior to 2006 \\
\hline $\begin{array}{c}\text { Published in English } \\
\text { language }\end{array}$ & $\begin{array}{c}\text { Published in language other } \\
\text { than English }\end{array}$ \\
\hline $\begin{array}{c}\text { Related to sexual } \\
\text { addiction } \\
\text { controversy topic }\end{array}$ & $\begin{array}{c}\text { Did not related to sexual } \\
\text { addiction controversy topic }\end{array}$ \\
\hline Full text available & Full text not available \\
\hline
\end{tabular}

Based on the inclusion and exclusion criteria that had been determined, the researchers identified 24 electronic journal articles. After going through review process, 12 articles were selected as those articles were the most relevant to the research topic.

\section{RESULTS}

The results are divided into two themes. The first one is a group of pro arguments that support sexual addiction to be classified as a mental disorder. The second one is a group of contra arguments that against sexual addiction to be classified as a mental disorder. So, how is the controversy over the status of sexual addiction as a mental disorder?

\subsection{Pro Arguments that Support Sexual Addiction to be Classified as A Mental Disorder}

1. The main argument that states that sexual addiction deserves to be categorized as psychological disorders is that sexual addiction is believed to have negative impacts on the life functions of individuals who experience it. The negative consequences of sexual addiction are similar to the consequences of other addictive disorders [1]. Both sexual addiction and other addictions can interfere with the life function of individuals who experience addiction, especially in areas of family life, professional life, and personal life. Or in other words, sexual addiction can cause psychosocial problems for those who experience it. In addition, there are also negative consequences caused by unprotected sexual intercourse that is usually done by someone who experiences sexual addiction, such as the risk of HIV, sexually transmitted diseases, and unwanted pregnancies [3, 4].

2. Sexual addiction is thought to have similar pattern to drug addiction where there is a phase of withdrawal between sexual episodes experienced. As many as $70 \%$ of patients with sexual addiction report that they experience withdrawal symptoms 
[1]. The symptoms of withdrawal are nervous, sweating, nausea, increased heart rate, shortness of breath, and fatigue [5].

3. Sexual addiction is formed through the same process with other addictions, that is through the process of experimentation or trial and error, using occasionally for pleasure, using regularly, until the stage of becoming dependent on a behavior or substance [6].

4. There are similarities in the brain parts of individuals with sexual addiction and individuals who experience substance addiction, where there is greater activation in the part of the brain associated with the reward system. However, there are still few studies that examine this, so that further studies on the neurological basis of sexual addiction are still needed [6].

5. There are patients who really complain about their high sexual urges and have difficulty in regulating their sexual behavior that cause significant negative problems and consequences [7]. Although until now there have been no large-scale epidemiological studies ever conducted, several studies have attempted to estimate the prevalence of sexual addiction. Most studies estimate that the prevalence of sexual addiction ranges from $3 \%$ to $6 \%$ of the adult population [1]. Moreover, there are complaints from mental health practitioners in the field who experience confusion when getting patients with symptoms of excessive and uncontrolled sexual behavior.

This confusion arises because there are patients who complain about the symptoms similar to sexual addiction but in the other hand the diagnosis of those symptoms can't be found in DSM, so psychologists often experience confusion about what treatments or interventions should be given to these patients. The confusion is mainly about whether they have to treat this as an addiction or not. Therefore, oftentimes psychologists don't focus the intervention on the excessive sexual behavior but instead focus the intervention on other disorders that arise, such as personality disorders, mood disorders, or others. Alexandra Katehakis Ph.D., MFT, a therapist who focuses on sexual dysfunction, sexual anorexia, sexual addiction and love addiction writes the impact of not including sexual addiction as a disorder in DSM-5 [8]. Katehakis states that the impact of not considering sexual addiction as a disorder is that it creates confusion among therapists in choosing the right treatment for clients and makes the treatments for sexual addiction become inaccessible to many people because if it is not included in the diagnosis, then it cannot be covered by insurance.

\subsection{Contra Arguments that Against Sexual Addiction to be Classified as a Mental Disorder}

1. There are several reasons that make sexual addiction is not classified as a disorder, especially those that cause sexual addiction not included in the DSM-5. The main reason came from the previous DSM editor, Robert Spitzer (DSM-III) and Allen Frances (DSM-IV), where they used the Psychiatric Times and other forums to express criticism about the inclusion of sexual addiction or hypersexual disorders in the DSM-5 [7]. It was stated that sexual addiction does not yet have sufficient scientific research to be enforced as a disorder, where there is not enough research on anatomical and functional imaging, molecular genetics, pathophysiology, epidemiology and neuropsychological testing. In line with this criticism, now in the DSM-5 it is explained that the refusal of sexual addiction to enter DSM- 5 because at this time peer-reviewed evidence is still not sufficient to enforce diagnostic criteria.

The addition of a diagnosis of a new disorder is always in tandem with the possibility of increasing false- positive diagnoses and the consequence of giving unnecessary strong psychotropic medications to individuals [9]. False-positive diagnoses occur when an individual is diagnosed with a disorder whereas in reality the individual does not have the disorder [10]. False positive diagnoses have the potential to occur in sexual addiction if a diagnosis of the disorder is made because issues related to sexual addiction are often confusing, whether sexual addiction is a health problem and a disorder or a problem that is actually more related to moral and social issues. Moreover, until now there are no adequate criteria to distinguish between normal and pathological levels of sexual desire and sexual activity [11]. Reference [12] mentioned that there are recent studies that show that many people who identify themselves as sex addicts are actually engaged in less sexual activity than average, but they feel more ashamed about their sexuality and have more negative attitudes toward sex. This can indicate that what we have referred to as sexual addiction all this time is actually not a disorder, but an inconvenience that arises in someone because they feel that their sexual desires are contrary to cultural or religiosity values which implies that high sexual desire is something that is inappropriate. 
2. Has the potential to be misused in the forensic context. If the diagnosis of sexual addiction is enforced, it is feared that it will be misused as an excuse to reduce the sentence of the accused who committed a crime, such as child sexual abuse [13].

3. One of the criteria for hypersexual disorders that were proposed to DSM-5 is "Repeatedly engaging in sexual behavior regardless of the risk of physical and emotional harm that might occur to self and others." [14]. According to Moser, this criterion is not appropriate to be used as a criterion of a disorder because based on the logic of this criterion, then scuba diving, mountain climbing, and driving fast on highways should also be considered as psychological disorders because all of them have dangerous risks to self [15]. In fact, being involved in desired and enjoyable activities regardless of the potential risk of harm is a normal thing to do. Furthermore, he concluded that the diagnosis proposed has the basis of wrong and inconsistent logic, incorrect criteria, historical inaccuracy, and a construct that has not been arranged properly [15].

4. If there is a diagnosis of sexual addiction or hypersexual disorder, it is feared that the diagnosis will make the variations of healthy sexual behavior to be considered as something pathological since it doesn't meet the deviation requirements [16]. Issues regarding sexuality are issues that cannot be separated from the values that exist in society. In conservative society or society with certain values of religiosity, there are rules that limit the types of acceptable sexual behavior. For example, Langstrom and Hanson in their study tried to define excessive sexual behavior by limiting that only high rates of impersonal sex (e.g. masturbation, pornographic use, casual sex, multiple partners, and sex groups) that can be defined as excessive sexual behavior, while high rates of personal sex (sexual intercourse in romantic relationships) won't be defined as excessive [17]. This shows that the limits of whether or not sex behavior considered as excessive is not only determined by the frequency or duration of sex, but also related to the types of sexual behavior that is accepted by certain values in society. This can also indicate the existence of a double standard that seems to make certain types of sexual activities that are considered as incompatible with certain values to be considered as abnormal or even pathological sexual behaviors, even though it does not necessarily have negative physical or psychological impacts.
5. One of the most often criticisms conveyed by those who against the inclusion of sexual addiction to DSM-5 is that sexual addiction or hypersexual disorders can be a form of manifestation of other mental disorders [13, 15]. This criticism is very important to be noticed because the patients who complain of hypersexuality often have comorbidities with other mental disorders. Conditions that reflect sexual addiction are often comorbid with psychiatric disorders such as mood disorders, social phobia, anxiety disorders, dysthymia, impulsivity, deficit hyperactivity disorder (ADHD) [18], and post-traumatic stress disorder (PTSD) [5].

6. Many clinicians believe that patients who complain of hypersexuality usually use sex as a coping mechanism when they are in difficult situations or use sex as a coping mechanism when experiencing emotional distress [7]. In other words, hypersexuality is an attempt to compensate a maladaptive system designed to regulate emotions. For example, when experiencing anxiety, depression, or loneliness that is felt to be very disturbing, a person does sexual behavior to temporarily relieve the negative emotions. Then a question arises, is hypersexuality or what we commonly call as sexual addiction an emotional dysfunction/adjustment disorder or an independent disorder? If we want to consider sexual addiction or hypersexual disorder as an independent disorder, it is necessary to identify which internal functions fail to operate and make a theory that explains what mechanism of action causes the failure of the function [7]. However, until now, no researcher has succeeded in identifying this [19].

\section{CONCLUSION}

To answer the question about whether sexual addiction is a mental disorder or not, firstly we need to know what a mental disorder is. Mental disorder is a set of symptoms caused by a dysfunction that occurs in individuals [20]. To determine something as a mental disorder, it is important to find the negative consequences and failure of a mechanism to carry out its biological functions. The biggest barrier to consider sexual addiction as a disorder is that there is no conceptual model and diagnostic criteria that can distinguish between normal sexual drive and activity with the pathological ones. Even if there are some attempts to distinguish them, the criteria still seem blurry and unclear. Even after Kafka reviews the evidence, there is no distinct bimodal distribution or taxon found to effectively define excessive sexual behavior [14]. DSM-5 website actually has tried to present rationale to consider a dysfunction that might 
occur because of hypersexual disorder. The models proposed are sexual desire/arousal dysregulation, sexual addiction, and sexual compulsivity. However, these models are still conceptually blurry because they don't provide any evidence of sexual desire dysfunction or abnormality [11]. Thus, until now, there has been no dysfunction caused by sexual addiction that can be clearly identified. Moreover, sexual addiction can be a manifestation of high sexual desire that becomes a problem because an individual try to manage it to be in accordance with the existing cultural and sexual norms as this condition is very subjective and value laden [21]. Therefore, it can be assumed that actually sexual addiction may not a disorder but an inconvenience that appears in individuals because of the influence of the culture that implies a value that having a high sexual desire is something that is inappropriate, and thus giving rise to feelings of guilt and remorse in people who have high sexual desires.

Besides, the lack of scientific evidence that shows a clear boundary between normal and pathological drives and sexual activity is very important to note, because if we classify sexual addiction as a mental disorder without strong scientific evidence, then this will potentially cause some negative consequences. The negative consequences that might arise are increasing false-positive diagnoses, being misused in the context of forensic, and potential to pathologize the healthy variations of sexual behaviors.

According to the literature review that has been conducted, the inclusion of symptoms similar to sexual addiction with the term Compulsive Sexual Behavior Disorder (CSBD) on the ICD-11 is actually departed from scientific evidence that is still being developed. Some reasons for the inclusion of CSBD on ICD-11 are to be able to improve the consistency of diagnosis and treatment carried out by professionals and also to reduce the shame and guilt of CSBD patients who want to seek help. In other words, the inclusion of CSBD on ICD-11 does not necessarily mean that CSBD already has a very strong scientific foundation.

So, based on the literature review that has been done, the label "sexual addiction" as a mental disorder is a term that is still premature and dangerous to be given, especially because the scientific limits underlying its diagnosis are still unclear and inconsistent. Another reason is due to the fact that other psychological problems and emotional regulation problems often cooccur in patients who have excessive sexuality problems so there is a possibility that what we have known so far as sexual addiction is actually a manifestation of other mental disorders and is not an independent disorder.

\section{REFERENCES}

[1] Karila, L., Wery, A., Weinstein, A., Cottencin, O., Petit, A., Reynaud, M., \& Billieux, J. (2014). Sexual Addiction or Hypersexual Disorder: Different Terms for the Same Problem? A Review of the Literature. Current Pharmaceutical Design, 20(25), 4012-4020 doi:10.2174/13816128113199990619

[2] American Psychiatric Association. (2013). Diagnostic and Statistical Manual of Mental Disorders, Fifth Edition. Arlington, VA: American Psychiatric Publishing.

[3] Carnes, P. J. (2000). Sexual Addiction and Compulsion: Recognition, Treatment, and Recovery. CNS Spectrums, 5(10), 6374.doi:10.1017/s1092852900007689

[4] Parsons, J. T., Grov, C., \& Golub, S. A. (2012). Sexual Compulsivity, Co-Occurring Psychosocial Health Problems, and HIV Risk Among Gay and Bisexual Men: Further Evidence of a Syndemic. American Journal of Public Health, 102(1), 156-

\section{2.doi:10.2105/ajph.2011.300284}

[5] Carnes P. (1991). Don't Call It Love. New York, NY: Bantam Books

[6] Estellon, V., \& Mouras, H. (2012). Sexual addiction: insights from psychoanalysis and functional neuroimaging. Socioaffective Neuroscience \& Psychology,

2(1), 11814 .

doi:10.3402/snp.v2i0.11814

[7] Reid, R. C., \& Kafka, M. P. (2014). Controversies About Hypersexual Disorder and the DSM-5. Current Sexual Health Reports, 6(4), 259-264. doi:10.1007/s11930-014-0031-9

[8] Sex Addiction Beyond the DSM-V. (n.d.) Retrieved from https://www.psychologytoday.com/intl/blog/sexlies- trauma/201212/sex-addiction-beyond-thedsm-v

[9] Phillips, J., Frances, A., Cerullo, M. A., Chardavoyne, J., Decker, H. S., First, M. B., ... Zachar, P. (2012). The six most essential questions in psychiatric diagnosis: a pluralogue part 1: conceptual and definitional issues in psychiatric diagnosis. Philosophy, Ethics, and Humanities in Medicine, 7(1), 3. doi:10.1186/1747-5341-7-3

[10] Spitzer, Robert, L., \& Wakefield, J. C. (1999). DSM-IV diagnostic criterion for clinical significance: Does it help solve the false positives 
problem? American Journal of Psychiatry 156: $1856-1864$.

[11] Wakefield, J. C. (2012). The DSM-5's proposed new categories of sexual disorder: the problem of false positives in sexual diagnosis. Clin Soc Work J., 40, 213-23.

[12] Sex Addiction. (n.d.). Retrieved from https://www.psychologytoday.com/intl/basics/sexaddiction

[13] Halpern, A. L. (2011). The Proposed Diagnosis of Hypersexual Disorder for Inclusion in DSM-5: Unnecessary and Harmful. Archives of Sexual Behavior, 40(3), 487-488. doi:10.1007/s10508011-9727-3

[14] Kafka, M. P. (2010). Hypersexual disorder: A proposed diagnosis for DSM-V. Archives of Sexual Behavior, 39, 377-400.

[15] Moser C. (2011). Hypersexual disorder: just more muddled thinking.

Arch Sex Behav, 40(2), 227-9.
[16] Giles, J. (2006). No Such Thing as Excessive Levels of Sexual Behavior. Archives of Sexual Behavior, 35(6), 641-642. doi:10.1007/s10508006-9098-3

[17] Långström, N., \& Hanson, R. K. (2006). High Rates of Sexual Behavior in the General Population: Correlates and Predictors. Archives of Sexual Behavior, 35(1), 3752.doi:10.1007/s10508-006- 8993-y

[18] Mick, T. M., \& Hollander, E. (2006). ImpulsiveCompulsive Sexual Behavior. CNS Spectrums, 11(12), 944-

955.doi:10.1017/s1092852900015133

[19] Van Lankveld, J. (2012). The road ahead: theoretical models to guide new sex research. J Sex Res., 49(2-3), 103-4.

[20] Kingston, D. A. (2018). Hypersexuality: Fact or Fiction? The Journal of Sexual Medicine, 15(5), 613-615. doi:10.1016/j.jsxm.2018.02.015।

[21] Levine, M. P., \& Troiden, R. R. (1988). The myth of sexual compulsivity. Journal of Sex Research, 25(3),

347-363. 